\title{
MODULES OVER SUBALGEBRAS OF THE DISK ALGEBRA
}

\author{
VERN I. PAULSEN AND DINESH SINGH
}

\begin{abstract}
This paper deals with the problem of characterizing submodules of $\mathbf{C}(\mathbf{T})$ over certain subalgebras of the disk algebra $\mathbf{A}$. We obtain results that are analogues of the classical characterizations of subspaces of $\mathbf{C}(\mathbf{T})$ that are invariant under multiplication by $z$, i.e., that are submodules over $\mathbf{A}$. These characterizations yield generalizations of Wermer's maximality theorem applicable to these subalgebras. We also present an invariant subspace theorem that seems to be of independent interest and show the equivalence of the classical theorem of the brothers Riesz on analytic measures with the theorem of Fatou.
\end{abstract}

\section{INTRODUCTION}

This paper deals ${ }^{1}$ with the problem of characterising submodules of the algebra of continuous functions on the circle, $\mathbf{C}(\mathbf{T})$, over certain subalgebras of the disk algebra, A.. Besides other outcomes, such a problem in our situation, as explained below, generalizes classical results such as Wermer's maximality theorem. Both these classical results have been tackled in various ways but the approach of Helson, [4], [5], seems to have been the most fruitful in enabling generalisations and extensions in several directions. Our methods also stem from the ideas of Helson, [4], [5] and are in the spirit of the circle of ideas that centre around the Helson-Lowdenslager Theorem. Specifically, we tackle the following two problems: $(i)$ characterizing those submodules of $\mathbf{C}(\mathbf{T})$ over subalgebra of the disk algebra that are constant on a set of measure $0,(i i)$ characterising submodules of $\mathbf{C}(\mathbf{T})$ over the subalgebra $\mathbf{A}_{1}=\left\{f \in \mathbf{A}: f^{\prime}(0)=0\right\}$ that contain $\mathbf{A}_{1}$.

Both problems are directly inspired by the maximality theorem of Wermer, which says that the disk algebra is maximal among all proper subalgebras of $\mathbf{C}(\mathbf{T})$.. One way that Wermer's theorem can be proved is by noting that any algebra that contains the disk algebra is necessarily a module over the disk algebra. A result of Hasumi-Srinivasan[3] characterizes the proper submodules of $\mathbf{C}(\mathbf{T})$ that are modules over the disk algebra. By examining

\footnotetext{
Date: October 15, 2004.

${ }^{0} 2000$ Mathematics Subject Classification Primary 46J15; Secondary 46E15, 46E25, $47 \mathrm{~J} 15$

${ }^{0}$ Key words and phrases. submodules over A, closed ideals, Wermers maximality theorem, invariant subspaces.

${ }^{1}$ The work of the first author is supported in part by the NSF
} 
which of these modules can also be algebras, one quickly obtains Wermer's theorem. Thus, the primary goal of this paper is to obtain generalizations of the Hasumi-Srinivasan result for modules over these two types of subalgebras. For the first type we then use the characterizations to obtain some extensions of Wermer's theorem.

The second subalgebra is an example of the algebras being studied by Agler and McCarthy[1] and are related to function theory on "embedded disks". We explain this comment more fully in Section 3. Along the way we present a new result on invariant subspaces of the algebra $\mathbf{A}_{1}=\{f \in$ $\left.\mathbf{A}: f^{\prime}(0)=0\right\}$ acting on the Hardy spaces and we use this result to characterize the closed submodules of $\mathbf{C}(\mathbf{T})$ over $\mathbf{A}_{1}$.

In [10] Srinivasan and Wang have established the equivalence of several fundamental results in the context of Hardy spaces on weak star Dirchlet algebras. However the following two classical theorems do not figure anywhere in their scheme of equivalent results: The well known F. and M. Riesz theorem about analytic measures and the theorem of Fatou about the existence, for each set $\mathbf{k}$ of Lebesgue measure zero, of a function in $\mathbf{A}$ that vanishes precisely on $\mathbf{k}$. These two results are the primary results that we use in our proofs and we prove that the two results are equivalent.

Acknowledgement: The second author would like to acknowledge the help and support of the Department of Mathematics of the University of Houston and also of the Mathematical Sciences Foundation, St. Stephen's College, Delhi.

\section{NOTATION AND TERMINOLOGY.}

Let $\mathbf{T}$ stand for the unit circle in the complex plane and let $\mathbf{C}(\mathbf{T})$ be the Banach algebra, under the supremum norm, of all continuous functions on T. Let $m$ stand for normalised Lebsgue measure on the unit circle. The disk algbera $\mathbf{A}$ consists of all functions in $\mathbf{C}(\mathbf{T})$ whose extensions to the open unit disk $\mathbf{D}$ are holomorphic. The algebra of all bounded holomorphic functions on $\mathbf{D}$ is denoted by $\mathbf{H}^{\infty}$. On the circle, $\mathbf{H}^{\infty}$ can also be viewed as the weak star closure of $\mathbf{A}$ in the algebra $\mathbf{L}^{\infty}$ of all essentialy bounded functions on $\mathbf{T}$ when $\mathbf{L}^{\infty}$ is treated as the dual of $\mathbf{L}^{1}$, i.e., $\mathbf{L}^{1}(d m)$. Given a subset $\mathbf{M} \subseteq \mathbf{L}^{\infty}$, we let $\operatorname{ann}(\mathbf{M})$ denote the set of measures on $\mathbf{T}$ that annihilate $\mathbf{M}$ and we let $\mathbf{M}_{\perp} \subseteq \mathbf{L}^{\mathbf{1}}$ denote its preannihilator. Let $\rho$ be the multiplicative linear functional on A given by $\rho(f)=f(0)$ so that the representing measure for $\rho$ is $m$ (normalised Lebesgue measure) on $\mathbf{T}$. We denote by $\mathbf{A}_{0}$ the kernel of $\rho$ and by $\mathbf{H}_{0}^{\infty}$ the weak star closure of $\mathbf{A}_{0}$ in $\mathbf{H}^{\infty}$. Since $\mathbf{A}$ is a subalgebra of $\mathbf{C}(\mathbf{T})$ there is a natural module action of $\mathbf{A}$ on $\mathbf{C}(\mathbf{T})$. A proper non-zero norm closed subspace of $\mathbf{C}(\mathbf{T})$ will be called invariant under multiplication by the co-ordinate function $z$ if and only if it is a module over $\mathbf{A}$. Such a subspace will be called an $\mathbf{A}$ submodule over $\mathbf{A}$. For any closed subalgebra $\mathbf{N}$ of $\mathbf{A}$, a closed subspace $\mathbf{M}$ of $\mathbf{C}(\mathbf{T})$ will be called an $\mathbf{N}$ module if $f g \in \mathbf{M}$ for all $f$ in $\mathbf{N}$ and all $g$ in $\mathbf{M}$. A proper non-zero closed subspace $\mathbf{M}$ of $\mathbf{L}^{p}(d m)$ is 
said to be doubly invariant if $z \mathbf{M}=\mathbf{M}$. and it is said to be simply invariant if $z \mathbf{M} \varsubsetneqq \mathbf{M}$. Simple invariance is equivalent to $\mathbf{A}_{0} \mathbf{M}$ i.e. $\left\{f g: f \in \mathbf{A}_{0}\right.$ and $g \in \mathbf{M}\} \varsubsetneqq \mathbf{M}$ as well as to $\mathbf{H}_{0}^{\infty} \mathbf{M} \varsubsetneqq \mathbf{M}$. A closed subspace $\mathbf{M}$ of $\mathbf{L}^{p}$ shal be called invariant under a subalgebra $\mathbf{B}$ of $\mathbf{C}(\mathbf{T})$ if $\mathbf{B M} \subset \mathbf{M}$. The space of all functions in $\mathbf{L}^{p}$ that have an analytic Fourier series shall be denoted by $\mathbf{H}^{p},(1 \leq p \leq \infty)$. A function $\phi$ in $\mathbf{H}^{p}$ that satisfies $|\phi|=1$ a.e. on $\mathbf{T}$ shall be called an inner function. An outer function in $\mathbf{H}^{p}$ is a function $\psi$ such that $\{\psi p\}$ where $p$ runs through all polynomials in $\mathbf{H}^{p}$ is norm dense in $\mathbf{H}^{p}$. For all facts and results pertaining to the Hardy spaces etc. we refer to $[2],[5],[6]$ and $[7]$.

\section{Statement OF MAin RESUlts}

Our main results are Theorem 3.1 and Theorem 3.6. We defer their proofs until Section 5 and instead here we focus on their statements and consequences.

Given a closed set $\mathbf{w} \subset \mathbf{T}$, we set $\mathbf{Z}(\mathbf{w})$ denote the ideal of functions in $\mathbf{C}(\mathbf{T})$ that vanish on $\mathbf{w}$ and we set $\mathcal{I}(\mathbf{w})=\mathbf{A} \cap \mathbf{Z}(\mathbf{w})$. Note that $\mathcal{I}(\mathbf{w})=(\mathbf{0})$ unless $m(\mathbf{w})=\mathbf{0}$. We let $\mathcal{A}(\mathbf{w})=\mathbb{C}+\mathcal{I}(\mathbf{w})$ denote the subalgebra of the disk algebra obtained by adjoining the identity. When $\mathbf{w}$ is the empty set, we have that $\mathcal{A}(\mathbf{w})=\mathcal{I}(\mathbf{w})=\mathbf{A}$.

Given any set $\mathbf{M} \subseteq \mathbf{C}(\mathbf{T})$, we let $\mathcal{Z}(\mathbf{M})$ denote the set of common zeroes of functions in $\mathbf{M}$.

Theorem 3.1. Let $\mathbf{w} \subset \mathbb{T}$ be a closed set of measure 0 , and let $\mathbf{M}$ be a closed subspace of $\mathbf{C}(\mathbf{T})$ with $\mathcal{Z}(\mathbf{M})=\mathbf{k}$ such that $\mathbf{M}$ is a module over $\mathcal{A}(\mathbf{w})$, with $\mathbf{w} \subseteq \mathbf{k}$. If $\mathbf{M} \neq \mathbf{Z}(\mathbf{k})$, then $\mathbf{M}_{\perp}=\phi \mathbf{H}^{\mathbf{1}}$ and $\mathbf{M}=\bar{\phi} \mathbf{H}_{0}^{1} \cap \mathbf{Z}(\mathbf{k})$ where $\phi$ is a function with $|\phi|=1$, almost everywhere.

Clearly, by setting $\psi=z \bar{\phi}$, we may write $\mathbf{M}=\psi \mathbf{H}^{\mathbf{1}} \cap \mathbf{Z}(\mathbf{k})$ and $\mathbf{M}_{\perp}=$ $\bar{\psi} \mathbf{H}_{\mathbf{0}}^{\mathbf{1}}$.

Corollary 3.2. Let $\mathbf{k} \subseteq \mathbf{T}$ be a set of measure 0. Then $\mathcal{I}(\mathbf{k})$ is maximal among all proper, closed subalgebras of $\mathbf{Z}(\mathbf{k})$.

Proof. Assume that there is a closed subalgebra $\mathbf{B}$, such that, $\mathcal{I}(\mathbf{k}) \subseteq \mathbf{B} \subsetneq$ $\mathbf{Z}(\mathbf{k})$. Clearly $\mathbf{B}$ is a module over $\mathcal{I}(\mathbf{k})$ with $\mathcal{Z}(\mathbf{B})=\mathbf{k}$ and so by the theorem, $\mathbf{B}=\phi \mathbf{H}^{\mathbf{1}} \cap \mathbf{Z}(\mathbf{k})$ and $\mathbf{B}_{\perp}=\bar{\phi} \mathbf{H}_{\mathbf{0}}^{\mathbf{1}}$. Since $\mathbf{B}$ is an algebra $\mathbf{B} \cdot \mathbf{B}_{\perp} \subseteq \mathbf{B}_{\perp}$ and hence for every $b \in \mathbf{B}$ we have that $b \bar{\phi} z \mathbf{H}^{\mathbf{1}} \subset \bar{\phi} z \mathbf{H}^{\mathbf{1}}$. This clearly implies that $b \in \mathbf{H}^{\mathbf{1}}$ and hence $\mathbf{B} \subset \mathbf{H}^{\mathbf{1}} \cap \mathbf{Z}(\mathbf{k})$, and the result follows.

Corollary 3.3. Let $\mathbf{k} \subset \mathbf{T}$ be a set of measure 0. Then $\mathcal{A}(\mathbf{k})$ is maximal among all proper, closed subalgebras of $\mathbb{C}+\mathbf{Z}(\mathbf{k})$.

Proof. Given any closed subalgebra $\mathbf{C}$ between these two algebras, set $\mathbf{B}=$ $\mathbf{C} \cap \mathbf{Z}(\mathbf{k})$ and apply the corollary above.

Corollary 3.4. (Wermer's Maximality Theorem). [1], [5]. A is maximal among the closed subalgebras of $\mathbf{C}(\mathbf{T})$. 
Corollary 3.5. ([2], Hasumi-Srinivasan, Theorem 2) Let $\mathbf{M}$ be a closed subspace of $\mathbf{C}(\mathbf{T})$ such that $z \mathbf{M} \subsetneq \mathbf{M}$ with $\mathcal{Z}(\mathbf{M})=\mathbf{k}$. Then there is a unique unimodular function $\phi$ such that $\mathbf{M}=\phi \mathbf{H}^{1} \cap \mathbf{Z}(\mathbf{k})$

Proof. Clearly, $\mathbf{M}$ is a module over A. Since $\mathbf{M}$ is a simply invariant subspace, $\mathbf{M} \neq \mathbf{Z}(\mathbf{k})$. Now apply the theorem.

Corollary 3.6. (Minimality Theorem) Let $\mathbf{M} \subset \mathbf{C}(\mathbf{T})$ be a $\mathcal{A}(\mathbf{k})$-submodule with $\mathbf{k} \subseteq \mathcal{Z}(\mathbf{M})$ and $\mathbf{M} \neq \mathbf{Z}(\mathbf{k})$. Then the following are equivalent:

(1) $\mathcal{I}(\mathbf{k}) \subseteq \mathbf{M}$,

(2) $\mathbf{M}=\bar{\phi} \mathbf{H}^{1} \cap \mathbf{Z}(\mathbf{k})$ for some (necessarily unique) inner function $\phi$.

Proof. First assume that 2) holds. We have that $\mathcal{I}(\mathbf{k})=\bar{\phi} \phi \mathbf{A} \cap \mathbf{Z}(\mathbf{k}) \subseteq$ $\bar{\phi} \mathbf{H}^{\mathbf{1}} \cap \mathbf{Z}(\mathbf{k}) \subset \mathbf{M}$.

Conversely, if 1) holds, then $\mathbf{k} \subseteq \mathcal{Z}(\mathbf{M}) \subseteq \mathcal{Z}(\mathcal{I}(\mathbf{k}))=\mathbf{k}$. Hence, $\mathcal{Z}(M)=$ $\mathbf{k}$ and so by the theorem, $M=\psi H^{1} \cap \mathbf{Z}(\mathbf{k})$. There is an outer function $g$ that vanishes exactly on $\mathbf{k}$ and is in A. Thus, $g \in M$ and hence, $g=\psi h$ for some $h \in H^{1}$, that necessarily vanishes on $\mathbf{k}$. Since $g$ doesn't vanish on the interior of the disk and $\bar{\psi}=h / g$ except on a set of measure 0 , we see that $\bar{\psi} \in H^{\infty}$ and the result follows.

Remark 3.7. Wermer's maximality theorem can be deduced as a consequence of the minimality theorem.

Recently, Agler and McCarthy[1] have begun studying function theory and analogues of Nevanlinna-Pick theory on embedded disks. The subalgebra $\mathbf{A}_{1}=\left\{f \in \mathbf{A}: f^{\prime}(0)=0\right\}$ is one such example. To see the connection consider the map $\phi: \mathbf{D}^{-} \rightarrow \mathbb{C}^{\mathbf{2}}$ defined by $\phi(z)=\left(z^{2}, z^{3}\right)$, let $\mathbf{X}=\phi\left(\mathbf{D}^{-}\right)$ denote the image and let $\mathbf{A}(\mathbf{X}) \subseteq \mathbf{C}(\mathbf{X})$ denote the uniformly closed subalgebra generated by the two coordinate functions. Then composition with $\phi$ induces an isometric isomorphism, $\phi^{*}: \mathbf{A}(\mathbf{X}) \rightarrow \mathbf{A}_{\mathbf{1}}$. We characterise the submodules of $\mathbf{C}(\mathbf{T})$ over $\mathbf{A}_{1}$.

Theorem 3.8. Let $\mathbf{M}$ be a closed subspace of $\mathbf{C}(\mathbf{T})$ such that $\mathbf{M}$ contains $\mathbf{A}_{1}$ and is a module over $\mathbf{A}_{1}$ but not over $\mathbf{A}$. Then either

$$
\mathbf{M}=\left\langle z^{k-2} g>_{\perp} \cap<\left(\alpha z^{k-2}+\beta z^{k-1}\right) I>_{\perp} \cap \overline{z^{k} I} \mathbf{H}_{0}^{\infty} \cap \mathbf{C}(\mathbf{T})\right.
$$

or

$$
\mathbf{M}=<\left(\alpha z^{k-2}+\beta z^{k-1}\right) I>_{\perp} \cap \overline{z^{k} I} \mathbf{H}_{0}^{\infty} \cap \mathbf{C}(\mathbf{T})
$$

where $k \geq 0$ is an integer and $\phi$ and $g$ are in $\mathbf{H}^{\infty}$, unique up to constant multiples, such that $\phi=(\alpha+\beta z) I, \alpha, \beta$ are scalars, $\alpha \neq 0,|\alpha| \geq|\beta|$ and $I$ is an inner function, $I(0) \neq 0, g=z^{2} \bar{B} I(1+(\beta / \alpha) z)^{-1}$ where $B=$ $(z+(\overline{\beta / \alpha}))(1+(\beta / \alpha) z)^{-1}$.

Remark 3.9. The statement of the theorem is meant to imply that, in general, $\mathbf{M}$ can be either of two spaces. However, in certain cases, $\mathbf{M}$ is necessarily the second space and these cases are enumerated in Remark 4.4. 


\section{Some Invariant Subspaces}

The three results in this section, on invariant subspaces, are important in their own right and the first two are well known. The first two shall be used in Theorem . The third will help characterize submodules of $\mathbf{C}(\mathbf{T})$ over the subalgebra $\mathbf{A}_{1}$.

Theorem 4.1. (Helson-Lowdenslager-Srinivasan). A simply invariant subspace of $\mathbf{L}^{p}$ has the form $\phi \mathbf{H}^{p}$ for some unimodular function $\phi$. The function $\phi$ is unique up to multiplication by a scalar.

Proof. See [4]

Theorem 4.2. (Wiener) A doubly invariant subspace in $\mathbf{L}^{p}$ has the form $\mathbf{I}_{E} \mathbf{L}^{p}$ where $\mathbf{I}_{E}$ is the characteristic function of the set $E$ of positive Lebesgue measure.

Proof. See [4].

Theorem 4.3. Let $\mathbf{N}$ be a closed subspace of $\mathbf{H}^{p},(0<p \leq \infty$; weak star closed when $p=\infty$ ) such that $\mathbf{A}_{1} \mathbf{N} \subset \mathbf{N}$ but $\mathbf{A N} \nsubseteq \mathbf{N}$. Then there exist scalars $\alpha \neq 0$ and $\beta,|\alpha| \geq|\beta|$, an inner function $I, I(0) \neq 0$ and a nonnegative integer $k$, such that for $\phi=(\alpha+\beta z) I$ and $g=z^{2} \bar{B} I(1+(\beta / \alpha) z)^{-1}$ where $B=(z+(\overline{\beta / \alpha}))(1+(\beta / \alpha) z)^{-1}$, we have that $\mathbf{N}$ is either

$$
<z^{k} \phi>\oplus z^{k+2} I \mathbf{H}^{p}
$$

or

$$
<z^{k} g>\oplus<z^{k} \phi>\oplus z^{k+2} I \mathbf{H}^{p}
$$

Remark 4.4. Note that both of the above subspaces are always invariant under $\mathbf{A}_{\mathbf{1}}$. The proof of the theorem shall also indicate that $g$ is definitely zero and, consequently, $N$ is unique if any one of the following is true: $(i)$ $\beta=0$ or (ii) $\beta \neq 0$ but $I \equiv 1$ or (iii) $B \nmid I$.

Proof. The case $\mathbf{p}=\mathbf{2}$. We may write $\mathbf{N}=\mathbf{z}^{\mathrm{k}} \mathbf{M}$ where $\mathbf{M}$ has at least one function that does not vanish at the origin and this determines $k$ uniquely. The problem is trivial if $1 \in \mathbf{M}$, since in this case, $\mathbf{M}$ can easily be seen to be equal to $\mathbf{H}_{1}^{2}=\left\{f \in \mathbf{H}^{2}: f^{\prime}=0\right\}=<1>\oplus z^{2} \mathbf{H}^{2}$. Thus, we assume that $1 \notin \mathbf{M}$. Note that in this case, $1 \notin \mathbf{M}^{\perp}$. For if not, then, each function in $\mathbf{M}$ would vanish at the origin contradicting our first assumption. Hence we conclude that

$$
1=\phi+1-\phi, \quad \phi \in \mathbf{M}, 1-\phi \in \mathbf{M}^{\perp}
$$

with $\phi \neq 0$. Now, as $z^{n} \phi \in \mathbf{M}, \mathrm{n}=0,2,3, \ldots$, we observe that

$$
\int_{\mathbf{T}} z^{n} \phi(1-\bar{\phi}) d m=0, \quad n=0,2,3 \ldots
$$

This means that when $n=0$,

$$
\int_{\mathbf{T}} \phi d m=\int_{\mathbf{T}}|\phi|^{2} d m
$$


and when $n \geq 2$,

$$
\int_{\mathbf{T}} z^{n} \phi d m=\int_{\mathbf{T}} z^{n}|\phi|^{2} d m
$$

so that from (1) and (2) respectively we have

$$
0<\phi(0)<1
$$

and

$$
\int_{\mathbf{T}} z^{n}|\phi|^{2} d m=0, \quad n= \pm 2, \pm 3, \ldots
$$

Consequently,

$$
|\phi|^{2}=a \bar{z}+b+c z
$$

where $b \neq 0$ because of (1). It now follows by the Riesz-Fejer Theorem [8] that there exists a linear polynomial $\alpha+\beta z$ such that

$$
|\phi(z)|^{2}=|\alpha+\beta z|^{2}
$$

Since, we also have that $|\phi(z)|^{2}=|\bar{\beta}+\bar{\alpha} z|^{2}$, we assume without any loss of generality that $|\alpha| \geq|\beta|$. Now let $\phi$ be factorised as $I O$ where $I$ is its inner part and $O$ is its outer part. Since $\phi(0) \neq 0$, we have that $\alpha \neq 0$ and $I(0) \neq 0$. Also, since $|\alpha| \geq|\beta|$ we have that $\alpha+\beta z$ is an outer function and since $|O(z)|=|\phi(z)|=|\alpha+\beta z|$ by the uniqueness of the factorization up to a scalar, we may set

$$
O(z)=\alpha+\beta z
$$

and thus

$$
\phi(z)=(\alpha+\beta z) I(z)
$$

We also note that it may be that $\beta=0$ but in view of what has been said above $\alpha \neq 0$. We now claim that either

$$
\mathbf{M}=<g>\oplus<\phi>\oplus z^{2} I \mathbf{H}^{2}
$$

or

$$
\mathbf{M}=\left\langle\phi>\oplus z^{2} I \mathbf{H}^{2}\right.
$$

where $g$ is as given in the statement of the theorem.

Clearly, $\mathbf{M}_{1}=<\phi>\oplus z^{2} I \mathbf{H}^{p}$ is the smallest $\mathbf{A}_{\mathbf{1}}$-invariant subspace that contains $\phi$ and so is contained in $\mathbf{M}$. So what remains to be shown is that the orthogonal complement of $\mathbf{M}_{1}$ in $\mathbf{M}$ is at most the space $\langle g\rangle$. Towards this end let $h \in \mathbf{M} \ominus \mathbf{M}_{1}$. Then for $n=0,2,3, \ldots, z^{n} \phi \perp h$ and as $h$ lies in $\mathbf{M}, z^{n} h \perp 1-\phi$. These two orthogonal relations together yield, on taking $n=0, \int_{\mathbf{T}} h \bar{\phi} d m=0$ and $\int_{\mathbf{T}} h d m=\int_{\mathbf{T}} h \bar{\phi} d m$ so that

$$
h(0)=0 .
$$

also when $n=2,3, \ldots$, we have $\int_{\mathbf{T}} h \overline{\phi z^{n}} d m=0$ and $\int_{\mathbf{T}} h \bar{\phi} z^{n}=0$ so that

$$
h \bar{\phi}=\gamma \bar{z}+\eta z, \quad \quad \text { (for some scalars } \gamma, \eta \text { ) }
$$

Now, using the fact that $\phi=(\alpha+\beta z) I$ we get from (3) and (4)

$$
h(\bar{\alpha}+\bar{\beta} z)=\gamma \bar{z} I+\eta z I
$$


However, the L.H.S. of (5) has only non-negative Fourier coefficients because $h(0)=0$ but the R.H.S. of (5) has a negative Fourier coeficient since $I(0) \neq 0$ (because $\phi(0) \neq 0$ ). This can only mean that $\gamma=0$ so that

$$
h \bar{\phi}=\eta z .
$$

Now let there exist a $k$ in $\mathbf{M}$ such that $k \perp \mathbf{M}_{1}$. Then by the same reasoning as employed above to deduce (5) we conclude that

$$
k \bar{\phi}=\lambda z .
$$

(6) and (7) together yield

$$
(\lambda h-\eta k) \bar{\phi}=0 .
$$

However, non-zero $\mathbf{H}^{2}$ functions cannot vanish on any set of positive measure so we are forced to conclude that $h=\delta k$ for some non-zero scalar $\delta$. Hence $\mathbf{M} \ominus \mathbf{M}_{1}$ is at most one dimensional.

We now derive the precise conditions under which $\mathbf{M} \ominus \mathbf{M}_{\mathbf{1}}=\mathbf{0}$. We let $g$ denote a typical function in this space.

(i) When $\beta=0$, i.e., when $\phi$ is a scalar multiple of an inner function. Then clearly $g=\gamma z \phi$, by (6) with $h=g$. But then this shall mean that $z \phi \in \mathbf{M}$ since $g$ is assumed to be in $\mathbf{M}$. However, then we have that $\mathbf{M}=\phi \mathbf{H}^{2}$ because of the nature of $\mathbf{M}$ and the nature of $\phi$. This means $\mathbf{M}$ is invariant under the action of $\mathbf{A}$ which violates our hypothesis. This can only mean that $g=0$ and so $\mathbf{M}=\langle\phi\rangle \oplus z^{2} I \mathbf{H}^{2}$.

(ii) When $\beta \neq 0$ but $I \equiv 1$. Then by $(6), g(\bar{\alpha}+\overline{\beta z})=\eta z$ and since $g(0)=0$ we get

$$
h(\overline{\alpha z}+\bar{\beta})=\eta
$$

where $g=z h$. This also leads to a contradiction, if $\eta \neq 0$, because $|\alpha| \geq|\beta|$ implies that $h \notin \mathbf{H}^{\mathbf{2}}$. Hence, once again, we conclude that $\gamma=0$ so that $g=0$. Thus $\mathbf{M}=<\phi>\oplus z^{2} I \mathbf{H}^{2}$.

(iii) When $\beta \neq 0, I \neq 1$ and $B$ does not divide $I$ where $B=(z+$ $\overline{\beta / \alpha}) /(1+(\beta / \alpha) z)$. Let $g=z \psi \sigma$ where $z \psi$ is the inner part of $g$ and $\sigma$ its outer part. Rewriting (6) in this factorised form, where $g=h$,

$$
z \psi \sigma(\bar{\alpha}+\overline{\beta z}) \bar{I}=\gamma z
$$

so that

$$
\psi \sigma(\bar{\alpha} z+\bar{\beta})=\gamma z I
$$

If $|\alpha|=|\beta|$ then the L.H.S. of (8) above has a non-trivial outer factor, whereas the R.H.S. is an inner function which is a contradiction unless $\gamma=0$ and, consequently, $g=0$. Hence we assume $|\alpha|>|\beta|$, so that $(z+\overline{\beta / \alpha})$ has a zero in the open unit disc. Consequently we rewrite $(\bar{\alpha} z+\bar{\beta})$ as

$$
\begin{aligned}
& \frac{\bar{\alpha}(z+\overline{\beta / \alpha})}{1+(\beta / \alpha) z}(1+(\beta / \alpha) z) \\
= & \bar{\alpha} B(1+(\beta / \alpha) z)
\end{aligned}
$$


In view of this $(8)$ becomes

$$
\bar{\alpha} \psi \sigma B(1+(\beta / \alpha) z)=\gamma z I,
$$

so, if both sides are non-zero, then by the uniqueness of the inner-outer factorisation

$$
\psi B=\mu z I
$$

for some scalar $\mu$ of modulus one. Since $B(0) \neq 0$, this would imply that $B$ divides $I$, which is a contradiction because $B$ does not divide $I$. Hence $\gamma=0$ so that $g=0$ and so

$$
\mathbf{M}=<\phi>\oplus z^{2} I \mathbf{H}^{2} .
$$

We now demonstrate that besides under the above three conditions $g$ shall not be zero. So we are assuming that neither of $(i),(i i)$ or $(i i i)$ hold. This means that $\phi(z)=(\alpha+\beta z) I(z)$, where $\alpha \neq 0$ and $B$ divides $I$. In this context '(9) remains valid so that by (10), $\psi B=\gamma I$ or $z \psi=\gamma z \bar{B} I$ and $\sigma=$ $\bar{\alpha}^{-1}(1+(\beta / \alpha) z)^{-1}$. Then $g=\bar{\alpha}^{-1} \gamma z \bar{B} I(1+(\beta / \alpha) z)^{-1}$. By directly evaluating the integral $\int_{\mathbf{T}} g \bar{\phi} d m$ it is not too difficult to see that $g$ is orthogonal to $\phi$. Also since $(\alpha+\beta z)$ is an outer function $(\alpha+\beta z) \mathbf{H}^{2}$ is dense in $\mathbf{H}^{2}$ and hence $z^{2} \phi \mathbf{H}^{2}$ is dense in $z^{2} I \mathbf{H}^{2}$. Thus to show that $g$ is orthogonal $z^{2} I \mathbf{H}^{2}$ it is enough to show that $g$ is orthogonal $z^{2} \phi \mathbf{H}^{2}$. So for any $h \in \mathbf{H}^{2}$,

$$
\begin{aligned}
\int_{\mathbf{T}} g \overline{z^{2} \phi h} d m & =\int_{\mathbf{T}} z^{2} \bar{B}(1+(\beta / \alpha) z)^{-1} \overline{I z^{2}(1+(\beta / \alpha) z) I h} d m \\
& =\int_{\mathbf{T}} z^{2} \bar{B}(1+(\beta / \alpha) z)^{-1} \overline{I z^{2}(1+(\beta / \alpha) z) I h} d m \\
& =\int_{\mathbf{T}}(1+(\beta / \alpha) z)^{-1} \overline{B h} d m \\
& =\overline{B(-\bar{\beta} / \bar{\alpha}) h(-\bar{\beta} / \bar{\alpha})}=0
\end{aligned}
$$

because of the reproducing kernel $(1+(\beta / \alpha) z)^{-1}$.

To complete the proof of the theorem we must show that $\mathbf{M}$ has the same characterization even when $0<p \leq \infty$. Over here we only discuss the case $0<p<2$ which shall suffice for the proof of Theorem 2. The rest of the proof of the theorem along with related results and applications shall be published later in another note. The thing to observe is that if $\mathbf{M}$ is a nontrivial closed subspace of $\mathbf{H}^{p}$ invariant under $\mathbf{A}_{1}$ but not under $\mathbf{A}$ then the same is true for $\mathbf{M} \cap \mathbf{H}^{2}$. Of course the first thing that we have to show is that $\mathbf{M} \cap \mathbf{H}^{2}$ is non-trivial. So let $f$ be a non-zero function in $\mathbf{M}$. Let $f(z)=h(z)+z k(z)$ where the Fourier series of $h$ and $k$ consist of only even powers of $z$. Let

$$
h_{n}=\exp -\left(\frac{\sqrt{|h|}+i \widetilde{\sqrt{|h|}}}{n}\right) \exp -\left(\frac{\sqrt{|k|}+i \widetilde{\sqrt{|k|}}}{n}\right)
$$

then one can show, by steps similar to those in the proof of the main theorem of Paulsen-Singh[8], that $h_{n} f$ is actually in $\mathbf{M} \cap \mathbf{H}^{2}$ and that it converges in the $\mathbf{L}^{1}$ norm to $f$. This shows at once that $\mathbf{M} \cap \mathbf{H}^{2}$ is dense in $\mathbf{M}$. The 
density also implies that $\mathbf{M} \cap \mathbf{H}^{2}$ cannot be invariant under $\mathbf{A}$ and since it isobviously invariant under $\mathbf{A}_{1}$ the $\mathbf{H}^{2}$ version of the theorem yields at once the sought characterization of $\mathbf{M}$.

\section{Proofs of the main Results.}

We begin with the proof of Theorem 3.1.

Proof. Let $\mu$ be a measure that is in $\operatorname{ann}(\mathbf{M})$, the annihilator of $\mathbf{M}$. Let

$$
d \mu=f d m+d \nu_{S},
$$

be the Lebesgue decomposition of $\mu$ where $m$ is Lebesgue measure and $v_{S}$ is singular and supported on $S$ with $m(S)=0$. Let $g$ be an outer function in $\mathbf{A}$ that vanishes precisely on $\mathbf{w}$. Hence, $z^{n} g \in \mathcal{A}(\mathbf{w})$ and so for any $h$ in $\mathbf{M}, z^{n} g h \in \mathbf{M}$. This means, by the F. and M. Riesz Theorem, that

$$
g h d \mu=l d m,
$$

for some $l$ in $\mathbf{H}_{0}^{1}$. By (1) and (2) we conclude that

$$
g h f=l \text {, }
$$

and

$$
g h d \nu_{S}=0
$$

By (4), $\nu_{S}$ is supported on the set of zeros of $g$ (i.e. w) and of $h$ for each $h$ in $\mathbf{M}$. This simply means that $\nu_{S}$ is supported on the union of the set $\mathbf{w}$ and the set $\mathbf{k}$ of common zeros of functions in $\mathbf{M}$. However, since $\mathbf{w} \subseteq \mathbf{k}$ the support of $\nu_{S}$ lies inside $\mathbf{k}$. Moreover, since the support of $\nu_{S}$ lies inside $\mathbf{k}$, we conclude that $\nu_{S}$ lies in $\operatorname{ann}(\mathbf{M})$. This also means that $f$ annihilates $\mathbf{M}$.

If $f=0$ for all annihilating measures $\mu$, then we would have that $\operatorname{ann}(\mathbf{M}) \subseteq$ $\{\nu: \operatorname{supp}(\nu) \subseteq \mathbf{k}\}$, which would imply that $\mathbf{Z}(\mathbf{k}) \subseteq \mathbf{M}$, contradicting $\mathbf{M} \neq \mathbf{Z}(\mathbf{k})$. Hence, there exist non-zero $f$ such that $f d m \in \operatorname{ann}(\mathbf{M})$. Further, due to (3),

$$
h f=\frac{l}{g}
$$

But, since $g$ is an outer function and since $l$ is in $\mathbf{H}_{0}^{1}$ we conclude that $\frac{l}{g}$ is also in $\mathbf{H}_{0}^{1}$. Hence

$$
f \mathbf{M} \subset \mathbf{H}_{0}^{1} .
$$

The immediate conclusion from (5) is that no non-zero function in $\mathbf{M}$ can vanish on a set of positive measure because no non-zero function in $\mathbf{H}^{1}$ can vanish on any set of positive measure. Thus the Lebesgue measure of $\mathbf{k}$ is zero. Recall

$$
\mathbf{M}_{\perp}=\left\{f \in \mathbf{L}^{1}: f d m \in \operatorname{ann}(\mathbf{M})\right\},
$$

Also, if $f \in \mathbf{M}_{\perp}$ then $z^{n} g f \in \mathbf{M}_{\perp}$ for eachnon-negative $n$. Thus, if any $l$ is in the the weak star closure, in $\mathbf{L}^{\infty}$, of $\left\{z^{n} g: n=0,1, \ldots\right\}$ then it is not difficult to see that $l \mathbf{M}_{\perp} \subset \mathbf{M}_{\perp}$ However, the weak star closure of $\left\{z^{n} g: n=0,1, \ldots\right\}$ is $\mathbf{H}^{\infty}$ since $g$ is an outer function. This means that the closed subspace $\mathbf{M}_{\perp}$ 
of $\mathbf{L}^{1}$ is invariant under multiplication by $z$. Also, if $\mathbf{M}_{\perp}$ were to be doubly invariant then each function in it would vanish on a fixed set of positive measure and that would mean that the $f$ above would also vanish on the same set of positive measure. This would contradict (5) since no non-zero element of $\mathbf{H}_{0}^{1}$ can vanish on any set of positive measure. This means that $\mathbf{M}_{\perp}$ is a simply invariant subspace of $\mathbf{L}^{1}$ and hence there is a unimodular function $\phi,[4]$, such that

$$
\mathbf{M}_{\perp}=\phi \mathbf{H}^{1},
$$

Thus, $\mathbf{M}$ annihilates $\phi \mathbf{H}^{1}$ and hence

$$
\mathbf{M} \subseteq \bar{\phi} \mathbf{H}_{0}^{1} .
$$

We are now in a position to show that

$$
\mathbf{M}=\bar{\phi} \mathbf{H}_{0}^{1} \cap \mathbf{Z}(\mathbf{k}) .
$$

By virtue of the definitions of $\mathbf{k}$ and by (8) it is obvious that $\mathbf{M} \subset \bar{\phi} \mathbf{H}_{0}^{1} \cap$ $\mathbf{Z}(\mathbf{k})$. To establish the reverse inequality we proceed as follows: Let $h$ be in the right hand side of (9). Let $\mu$ be a measure that annihilates $\mathbf{M}$. Then, as established above $f$, the absolutely continuous part of $\mu$ shall lie in $\operatorname{ann}(\mathbf{M}) \cap \mathbf{L}^{1}$ and hence, in view of (7), it shall annihilate $h$. As regards the singular part of $\mu$, it is supported on $\mathbf{k}$ by what has been established near the beginning of the proof of the theorem. Thus the singular part of $\mu$ also annihilates $h$. This completes the proof of the theorem.

We now turn our attention to the proof of Theorem 3.8.

Proof. Let $d \mu$ be in $\operatorname{ann}(\mathbf{M})$, the annihilator of $\mathbf{M}$, and let

$$
d \mu=f d m+d \nu
$$

be its Lebesgue decomposition where $d \nu$ is supported on a closed set of Lebesgue measure zero. Then

$$
\int_{\mathbf{T}} z^{n} z^{2} d \mu=0
$$

for $n=0,1, \ldots$. Thus, by the F. and M. Riesz theorem, there is a $k$ in $\mathbf{H}_{0}^{1}$ such that

$$
z^{2} d \mu=k d m
$$

However, as is evident from (1),

$$
z^{2} d \mu=z^{2} f d m+z^{2} d \nu
$$

so that from (3) and (4) we infer that

$$
z^{2} f=k,
$$

and

$$
d \nu=0,
$$

Now it is easy to see that (5) implies that

$$
\mathbf{M}_{\perp} \subset \bar{z}^{2} \mathbf{H}_{0}^{1},
$$


and (7) implies that $\mathbf{M}_{\perp}=\bar{z}^{2} \mathbf{N}$ where $\mathbf{N}$ is a closed subspace of $\mathbf{H}_{0}^{1}$. Also it is not difficult to see that $\mathbf{N}$ is invariant under $\mathbf{A}_{1}$ but not under $\mathbf{A}$. Thus by Theorem 4.3, $\mathbf{N}$ is either

$$
<z^{k} g>\oplus<z^{k} \phi>\oplus z^{k+2} I \mathbf{H}^{1}
$$

or the function $g$ can be taken to be 0 and hence

$$
\begin{aligned}
\mathbf{M}_{\perp} & =\bar{z}^{2}\left(<z^{k} g>\oplus<z^{k} \phi>\oplus z^{k+2} I \mathbf{H}^{1}\right) \\
& =<z^{k-2} g>\oplus<z^{k-2} \phi>\oplus z^{k} I \mathbf{H}^{1}
\end{aligned}
$$

This clearly implies that

$$
\mathbf{M}=\left\langle z^{k-2} g>^{\perp} \cap<\left(\alpha z^{k-2}+\beta z^{k-1}\right) I>^{\perp} \cap \overline{z^{k} I} \mathbf{H}_{0}^{\infty} \cap \mathbf{C}(\mathbf{T})\right.
$$

\section{The equivalence of the Theorems of F. \& M. Riesz and of FATOU}

The following two theorems are classical and extremely important in the development of the circle of ideas that apply to that aspect of harmonic analysis which deals with spaces of functions on the unit circle and with harmonic analysis in the context of general function algebras. The first of these, the theorem of the brothers Riesz, is also interesting because of the number of interesting proofs that it has engendered. We refer to [1], [3], [4], [5] and [6].

Theorem 6.1. (F. E M. Riesz). Let $\mu$ be a Borel measure on the unit circle such that $\int_{\mathbf{T}} z^{n} d \mu=0, n=1,2, \ldots$ Then $\mu$ is absolutely continuous with respect to Lebesgue measure.

Theorem 6.2. (Fatou). Let $\mathbf{k}$ be set of zero Lebesgue measure in $\mathbf{T}$. Then there is a function in $\mathbf{A}$ that vanishes precisely on $\mathbf{k}$.

Theorem 6.3. The F. $\&$ M. Riesz theorem is equivalent to Fatou's theorem.

Proof. We first show how the theorem of Fatou leads to a proof of the F. and M. Riesz theorem. Let $d \mu=f d m+d \mu_{S}\left(f \in \mathbf{L}^{1} d m, d \mu \perp d \mu_{S}\right)$ be the Lebesgue decomposition of $\mu$ with respect to $m$. Let $\mathbf{k}$ be the support of $\mu_{S}$. Since $\mathbf{k}$ is a closed set of Lebesgue measure zero on the circle $\mathbf{T}$, there exists an outer functon $g$ such that $g$ is zero on $\mathbf{k}$. Clearly then $g d \mu=g f d m$. Since $g$ is in $\mathbf{A}$ we see from the fact that $\int_{\mathbf{T}} z^{n} g d \mu=0$ that $\int_{\mathbf{T}} z^{n} g f d m=0$ for $n=1,2,3 \ldots$ Thus $g f$ is in $\mathbf{H}^{1}$. Let $g f=h$. Then $f=\frac{h}{g}$ is in $\mathbf{L}^{1}$. But $h$ is in $\mathbf{H}^{1}$ and hence from the fact that $g$ is outer we conclude the fact that $\frac{h}{g}$ i.e. $f$ is in $\mathbf{H}^{1}$. Thus $\int_{\mathbf{T}} z^{n} f d m=0$ for $n=1,2,3 \ldots$ and hence $\int_{\mathbf{T}} z^{n} d \mu_{S}=0$. We now assume without any loss of generality that $g=1+z g_{1}$. Now, since $g_{1}$ is a uniform limit of polynomials in $z$, we conclude that $\int_{\mathbf{T}} z g_{1} d \mu_{S}=0$. But then we get $\int_{\mathbf{T}} d \mu_{S}=0$ because $g d \mu_{S}=0$. In the same way, from $z^{-1} g d \mu_{S}=0$ we have that $\int_{\mathbf{T}} z^{-1} d \mu_{S}=0$. Using induction we have $\int_{\mathbf{T}} z^{n} d \mu_{S}=0$ for all integers $n$ so that $\mu_{S}=0$, which proves the first half of the theorem. 
We now show the reverse implication i.e. the route to Fatou's Theorem via the Riesz theorem. It is easy to construct a positive valued continuously differentiable function $u$ on $\mathbf{C}-\mathbf{k}$ that converges to $\infty$ on each point of $\mathbf{k}$ and such that $u$ is in $\mathbf{L}^{1}$. It then follows that $e^{-(u+i \tilde{u})}$ is continuous on $\mathbf{T}$ and vanishes precisely on $\mathbf{k}$. To complete the proof, all that remains is to show that $e^{-(u+i \tilde{u})}$ is in $\mathbf{A}$. So let $d \lambda$ be any measure that annihilates $\mathbf{A}$. and such that it does not annihilate $e^{-(u+i \tilde{u})}$. By the Riesz Theorem, $d \lambda=h d m$ for some $h$ in $\mathbf{H}^{1}$. Then by looking at the function $g_{r}\left(e^{i \theta}\right)=\exp -\left(u\left(r e^{i \theta}\right)+i \tilde{u}\right.$ $\left.\left(r e^{i \theta}\right)\right),(0<r<1)$, which converges boundedly pointwise to $e^{-(u+i \tilde{u})}$ almost everywhere and which is in $\mathbf{A}$ we conclude that $d \lambda$ annihilates $g_{r}$ for each $r$ and so it must also annihilate $e^{-(u+i \tilde{u})}$. We have a contradiction.

\section{REFERENCES}

[1] J. Agler and J. McCarthy, Lecture Notes from IWOTA 2004, Newcastle.

[2] J.B. Garnett, Bounded Analytic Functions, Academic Press, (1981)

[3] M. Hasumi and T. P. Srinivasan, Invariant subspaces of continuous functions, Can. Journal of Math. 17 (1965), 643-651

[4] H. Helson, Lectures on Invariant Subspaces, Academic Press, (1964)

[5] H. Helson, Harmonic Analysis, Hindustan Book Agency, (1995)

[6] K. Hoffman, Banach Spaces of Analytic Functions, Prentice Hall, (1962)

[7] P. Koosis, Introduction to $\mathbf{H}_{p}$ Spaces, Cambridge University Press, (1998)

[8] V. I. Paulsen and Dinesh Singh, A Helson-Lowdenslager-de Branges Theorem in $\mathbf{L}^{2}$, Proc. Amer. Math. Soc. 129(2000), 1097-1103.

[9] G. Polya and G. Szego, Problems and Theorems in Analysis II, Springer Verlag, (1971)

[10] D. Sarason, Algebras of functions on the unit circle, Bull. Amer. Math. Soc.79 (1973), 286-299

[11] T.P. Srinivasan and J. K. Wang, Weak*-Dirichlet algebras, Function Algebras (Ed. F. T. Birtel), Scot-Foresman, (1965), 216-249

DEPARTMENT OF MATHEMATICS, UNIVERSITY OF HOUSTON, HOUSTON, TEXAS 77204-3476, U.S.A.

E-mail address: vern@math.uh.edu

DEPARTMENT OF MATHEMATICS, UNIVERSITY OF DELHI, DELHI 10007, INDIA

E-mail address: dinesh_singh@hotmail.com

Vern Paulsen: Department of Mathematics, University of Houston, HousTON, TX 77204-3476 